\title{
Designing Adaptive Structures for Whole Life Energy Savings
}

\author{
Gennaro Senatore \\ EngD candidate, Civil Environmental \& Geomatic Engineering UCL - Expedition Engineering \\ Philippe Duffour \\ Dr., Civil Environmental \& Geomatic Engineering, UCL
}

Chris Wise

Prof., Civil Environmental \& Geomatic Engineering, UCL - Expedition Engineering

Pete Winslow

Dr., Expedition Engineering

Sean Hanna

Dr., Bartlett School of Architecture, UCL

\begin{abstract}
Designing structures with minimal environmental impact is emerging as a serious concern in the construction sector. Conventional structural design practice involves designing first for strength, followed by secondary checks on deflections and other serviceability limits states. If these limits are exceeded, the conventional solution has been to add material to increase stiffness. When the design is governed by unpredictable events such as fluctuating loads, strong wind storms or earthquakes, the structure is effectively overdesigned for most of its working life. This paper presents a methodology to design adaptive structures that minimize the whole life energy consumption. The methodology is illustrated on plane pin-jointed trusses, both determinate and indeterminate. Strategically placing actuators allow the internal flow of forces to be homogenized and displacements to be controlled. The actuators only start working when the loads reach a certain threshold. Below this threshold, the structure resists loads mainly passively thereby limiting significantly the operational energy used. It was found that both indeterminate and determinate topologies bring substantial energy savings up to $70 \%$ of the total energy.
\end{abstract}

\section{INTRODUCTION}

\subsection{Background and conceptual framework}

Active control has been used in civil engineering structures for a variety of purposes. The most widespread application so far has been in vibration control (Soong, 1988). Shape morphing has also been studied (Ramrakhyani, et al., 2005). The potential of using adaption to save material has been investigated by a few (Teuffel, 2004) but whether the energy saved by using less material makes up the energy consumed through control and actuation is a question that has so far received little attention. The methodology presented in this paper aims to address this. A design process is proposed that produces an optimum adaptive structure that minimizes the total energy spent throughout the whole life of the structure (embodied in the materials + operational).

The process is illustrated diagrammatically in the conceptual graph shown in Fig. 1. This graph shows the total energy as a function of some notional degree of active control of the structure. The whole life energy is made of two components: operational energy and the embodied energy. For a completely passive design the embodied energy (mass of material) dominates the whole life energy: members are designed to bear $100 \%$ of the maximum expected load to meet strength and servicea- bility requirements. By contrast for a highly adaptive design, the embodied energy will be small but the operational energy necessary to control and actuate throughout the life of the structure will be high. The methodology proposed here seeks the minimum in whole life energy that lies between these two extremes.

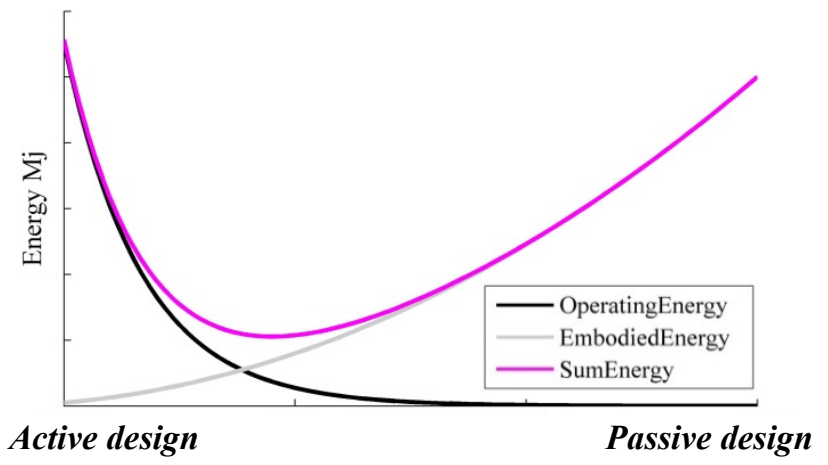

Figure 1 Whole-life energy vs. degree of adaptation

In a conventional design, members are sized based on a worst case scenario i.e. the maximum expected load combination. If embodied energy is to be saved, clearly, member sizing should not be governed directly by this worst load combination but by some fraction of it. As the loads approach their worst values, conventional members will reach their capacity. Then strategically located active elements provide controlled output energy (actuators) in order to manipulate actively the internal 
flow of forces and stresses. In this way stresses can be homogenized and deflections kept within desired limits. The active elements (actuators) are only activated for compensation of the displacements and internal forces when the loads reach a certain threshold. Therefore operational energy is only used when necessary.

It is intuitively clear that this design process will be particularly beneficial when the design is governed by large loading events that have a small probability of occurrence (storms, earthquakes, unusual crowds but also transient rolling loads such as trains). To illustrate this, Fig. 2 shows the cumulative frequencies of occurrence for a generic stochastic load. The pink line represents the activation threshold which demarcates two zones in the load range. On the left hand side are the more probable low levels of load that the passive load-bearing capacity of the structure will be able to withstand without actuation. On the right are rarer loads with higher magnitude which the structure will only be able to withstand using both passive and active load-bearing capacity.

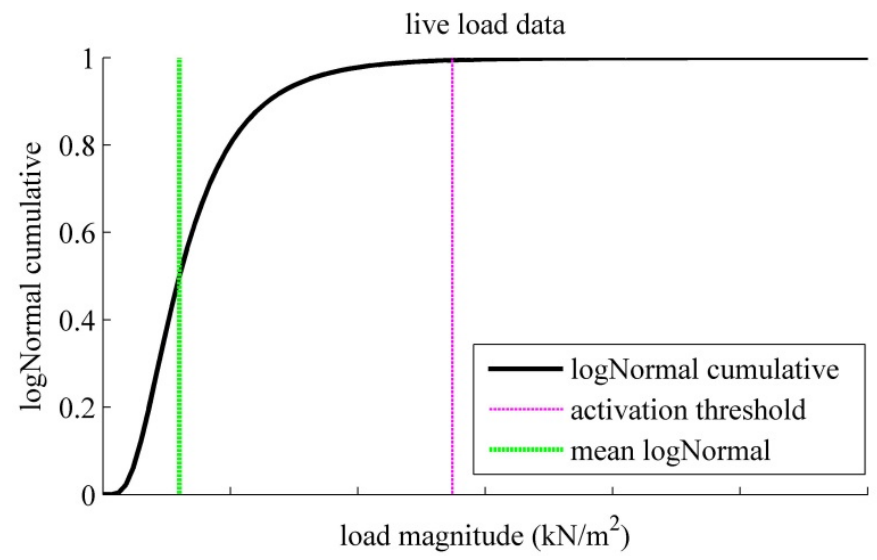

Figure 2 load activation threshold

\subsection{Case Study}

The design process is illustrated on a roof made of simple indeterminate cantilever planar trusses supporting 10 meters of cover each (out of plane). Fig. 3 shows the truss constrained at one end and subjected to both dead load and live load.

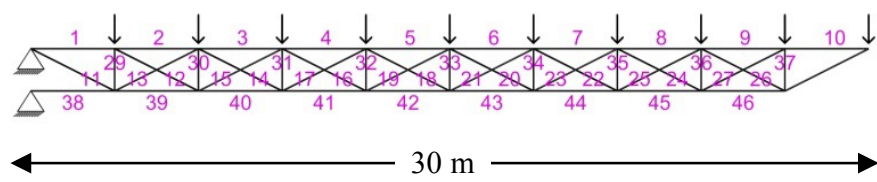

Figure 3 Cantilever pin-jointed truss

To test the effectiveness, the ratio of the cantilever's span to depth is deliberately set high at 25 (depth=1.2 m). The dead load on the roof panels is set to $3 \mathrm{kN} / \mathrm{m}^{\wedge} 2$ which means each truss supports a uniformly distributed load (UDL) of $30 \mathrm{kN} / \mathrm{m}$. The live load is modeled as a log-normal distribution with 0.5 standard deviation and mean at $20 \%$ of the max expected load. The load distribution acts on the structures for the $10 \%$ of structure's life-cycle assumed to be 50 years. For the purpose of this case study, the type of distribution can be considered representative of the majority of loading scenarios such as earthquake, strong winds, snow, rolling loads or even occupancy patterns in building. In terms of magnitude the maximum expected live load is set equal to the dead load.

\section{DESIGN METHODOLOGY}

\subsection{Load Path Optimization}

The first stage of the design process consists in minimizing the set of member cross-section areas $A_{i}$ and the bar forces $F_{i k}$ subjected to satisfying equilibrium constraints as well as a set of inequality constraints expressing that the member' forces are limited by material yield in tension and buckling in compression. At this stage, geometric compatibility is not yet respected. This initial phase of the methodology is inspired from Teuffel (Teuffel, 2004) but unlike Teuffel, the self-weight and buckling constraints are introduced making the problem nonlinear. The problem was solved using a nonlinear sequential quadratic solver (SQP) (Nocedal \& Wright, 2006). The problem can be formulated as follows:

$$
\begin{gathered}
\min V=\sum_{i=1}^{n} \mathrm{~A}_{i} * l_{i} \\
\text { s.t.: } \\
\boldsymbol{B} \cdot \boldsymbol{F}_{k}-\boldsymbol{P}_{k}=0 \\
\text { a) } \left.\frac{F_{i k}}{A_{i}} \leq \sigma_{T} \cdot M U T ; \quad b\right)-\frac{F_{i k}}{A_{i}} \leq \sigma_{C} \cdot M U T \\
\frac{F_{i k}}{A_{i}} \leq \sigma_{c r i t}
\end{gathered}
$$

where $V$ is the total volume, $l_{i}$ is the length of the $i^{\text {th }}$ element and $n$ is the total number of elements. Eq.2 is a set of equality constraints which represents the equilibrium equations: $\mathbf{B}$ is the cosine direction matrix and $k$ numbers the load cases. These constraints are nonlinear because the self-weight of the structure changes iteratively and so does the vector of external loads P. Ultimate limit states (ULS) are represented by the set of linear inequalities in eq. 3 where $\sigma_{\mathrm{T}}$ and $\sigma_{\mathrm{C}}$ are material yield stress in tension and compression respectively. Setting the material utilization factor (MUT) to 1, gives absolute minimum weight structures. The nonlinear stability constraints are represented in eq. 4 where critical loads $\left(\sigma_{\text {crit }}\right)$ are computed iteratively using the Euler buckling formulation. Eq. $3 \mathrm{~b}$ and eq. 4 are mutually exclusive for each element. 
Fig. 4 shows the optimal (non-compatible) distribution of axial forces and cross sectional areas obtained for the cantilever case study.

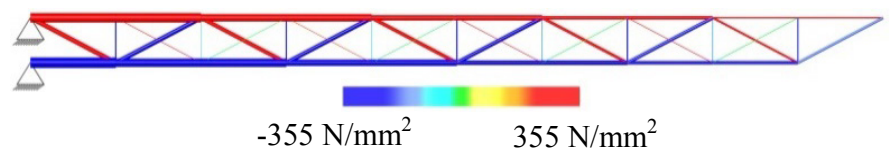

Figure 4 Optimal (non-compatible) forces distribution

The set of section areas is found by optimizing against the maximum expected load. Then a minimum set of bar forces must be found for any other instance of the load distribution. The problem can be formulated as:

$$
\left\{\begin{array}{c}
\text { a) } \min \left(\operatorname{norm}\left(\boldsymbol{F}_{\boldsymbol{k}}^{\boldsymbol{e q}}\right)^{2}\right) \\
\text { s.t. } \\
\text { b) } \boldsymbol{C}^{U L S+\text { buckling }} \cdot \boldsymbol{F}_{\boldsymbol{k}}^{\boldsymbol{e q}}-\boldsymbol{A}_{\boldsymbol{k}}^{\text {optimal }} \leq 0 \\
\text { c) } \boldsymbol{B} \cdot \boldsymbol{F}_{\boldsymbol{k}}^{\boldsymbol{e q}}=P_{\boldsymbol{j} \boldsymbol{k}}
\end{array}\right.
$$

where $\boldsymbol{F}_{\boldsymbol{k}} \boldsymbol{e q}$ is the optimal equilibrium pattern of forces for lower levels of loads than the maximum expected load; $\mathrm{C}^{\text {ULS+buckling }}$ is the matrix of the constraints from eqs(3-4) and $\mathrm{P}_{\mathrm{jk}}$ is $j^{\text {th }}$ component of load case $k$. If a determinate topology had been chosen for the truss, the actuator length changes would have only affected the geometry but not the state of stress of the structure (small displacements), i.e. $\boldsymbol{F}_{\boldsymbol{k}}{ }^{e q}$ is obtained by eq.5(c) directly.

Fig.5 shows the difference $\Delta \mathbf{F}_{\mathrm{k}}$ between the optimal (non-compatible) forces and the compatible axial forces of the structure without active control. The $\mathrm{x}$-axis shows the element numbers as labeled in Fig. 3 while vertical axis shows the force difference in $\mathrm{kN}$.

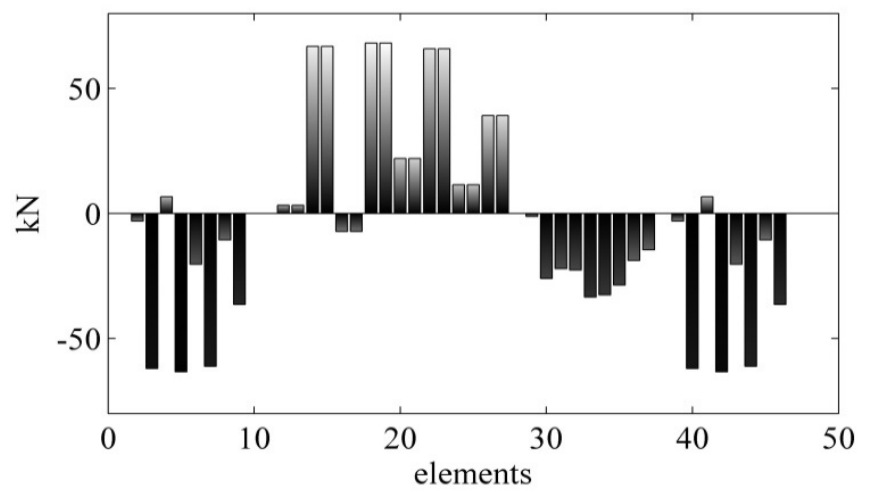

Figure 5 Difference between compatible and non-compatible axial force for each element.

As can be seen from Fig. 6, the maximum displacement is well beyond serviceability limits $(\operatorname{span} / 180=16 \mathrm{~cm})$

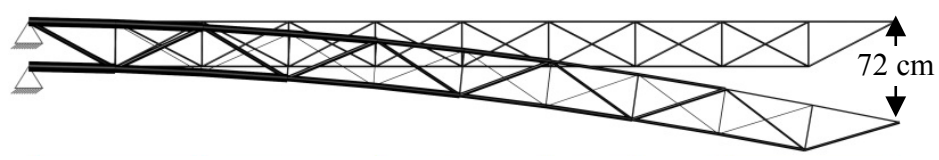

Figure 6 Compatible displacements without active control
For these reasons, active work in the form of actuator length change is needed for both forces and displacements correction.

\subsection{Active Element Topology}

The next stage in the design process involves finding the best location for the actuators. The optimum actuator positioning is found by first choosing a set of nodes whose displacement must be controlled (CDofs). For the truss studied here, the vertical displacements of all the nodes of the top chord are selected and constrained so that the cantilever tip moves within span/180 (i.e. $16 \mathrm{~cm}$ ). The difference between compatible and optimal forces $\Delta \mathbf{F}_{\mathbf{k}}$, and between the desired and compatible displacements $\Delta \mathbf{u}_{\mathbf{k}}$, are the inputs to find the most efficient (minimum operational energy) topology for the actuators.

The minimum desirable number of actuators is equal to the indeterminacy of the system plus the number of controlled DOFs which in this case is $8+9=17$. This is the minimum number of actuators necessary to turn the structure into a controlled mechanism. The most efficient positions for the actuators are those where the active elements have the largest effect on both axial forces and controlled displacements. This problem can be formulated as a least square optimization routine starting with the computation of the sensitivity matrices $\mathrm{S}^{\mathrm{U}}$ and $\mathrm{S}^{\mathrm{N}}$ for displacements and axial forces. These matrices store the effect of a unit length change for each element on nodal displacements (SU) and axial forces (SF) on the other elements. Each element length is increased by one unit length at a time. The resulting axial forces and nodal displacements are obtained using a flexibility approach (Patnaik, et al., 1991).

It is then possible to find the active element length changes $\Delta \mathrm{L}$ that satisfy the desired controlled DOFs $(\Delta \mathrm{u})$ eq. 6 and compatibility conditions eq.7:

$$
\begin{gathered}
\min \left\|\boldsymbol{S}^{\boldsymbol{U}} \cdot \Delta \boldsymbol{L}^{\text {all }}-\Delta \boldsymbol{u}\right\|^{2} \\
\boldsymbol{S}^{\boldsymbol{F}} \cdot \Delta \boldsymbol{L}^{\text {all }}=\Delta \boldsymbol{F}
\end{gathered}
$$

At this stage $\Delta \mathbf{L}_{\text {all }}$ is obtained considering all elements as active. In order to derive the set of most efficient elements the contribution towards the desired control displacement is evaluated as efficiency (8) of each member as:

$$
\begin{aligned}
\text { eff }_{i k} & =\frac{\sum_{i}^{n C D o f s} \frac{\boldsymbol{s}^{\boldsymbol{U}} \cdot \Delta \widetilde{\boldsymbol{L}_{\boldsymbol{k}}}}{\Delta \boldsymbol{u}_{\boldsymbol{k}}}}{n C D o f s} \\
\text { Eff }_{i} & =\frac{\sum_{k=1}^{\text {nloadCases }} \boldsymbol{e f f}_{\boldsymbol{k}}}{\text { nloadCases }^{\text {noad }}}
\end{aligned}
$$


where $\Delta \widetilde{L_{l k}}$ is the vector composed of the length change of element $\mathrm{i}$ for the load case $\mathrm{k}$ having all the others components set to 0 and nCDofs is the number of controlled Dofs. The global efficiency Eff $_{\mathrm{i}}$ of each member is obtained from eq. (9). Fig. 7 shows the optimum positioning of the actuators for our cantilever truss structure, given a free choice of their number and location.

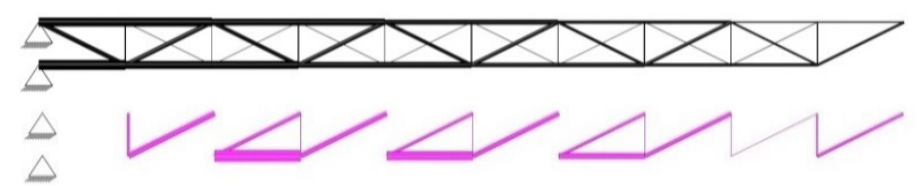

Figure 7 Optimal actuators topology

Once the position of the actuators is known, their required length changes is computed to bring the controlled nodes as close as possible to their required limits while enforcing the compatibility conditions eq.(11) as constraints:

$$
\begin{gathered}
\min \left\|\mathbf{S}_{\text {red }}^{U} \cdot \Delta \mathbf{L}-\Delta \mathbf{u}_{\text {red }}\right\|^{2} \\
\mathbf{S}_{\text {red }}^{\boldsymbol{F}} \cdot \Delta \mathbf{L}=\Delta \mathbf{F}
\end{gathered}
$$

$\mathbf{S}_{\text {red }} \mathbf{U}$ and $\mathbf{S}_{\text {red }}{ }^{\mathbf{F}}$ are reduced versions of $\mathbf{S}^{\mathbf{u}}$ and $\mathbf{S}^{\mathbf{F}}$ obtained by only keeping the columns corresponding to the chosen active elements. Finally, the length changes $\left(\Delta \mathrm{L}_{\mathrm{i}}\right.$ for each actuator as initial deformation) are imposed together with external loads to check that the displacements of the controlled DOFs are within the desired limits. Fig. 8 shows the comparison between the adaptive truss and the corresponding passive optimized version.

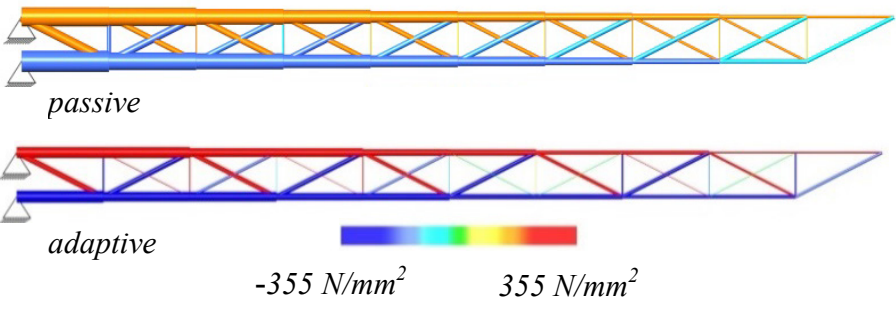

Figure 8 Optimal stress distribution passive vs. adaptive

\subsection{Embodied-operational energy optimization}

The work done by the actuators can be expressed as:

$$
W_{k}=\sum_{a c t T h r}^{\operatorname{size}\left(P_{k}\right)} \sum_{i}^{n a} \frac{\left(F_{i k}^{c o m p}+1 / 2 \cdot \Delta F_{i k}\right) \cdot \Delta L_{i k} \cdot f q \cdot t_{k}}{a c t M e c h E f f}
$$

In eq. (12) it is assumed that an actuator exerts a total force sum of $F_{i k}{ }^{c o m p}$, the passive element contribution with no active control (compatible), and additional force contribution $\Delta F_{i k}$ performing a length change $\Delta \mathrm{L}_{\mathrm{ik}}$. The operational frequency is kept constant for any occurrence time $e_{k}$ of each load case $P_{k}$ (hours of occurrence). Working frequency and working efficiency of the actuators are set as 5 $\mathrm{Hz}$ and $50 \%$ respectively as conservative assump- tions. The total operational energy is the sum of the energy needed for compensation of forces and displacements for all the loads above the activation threshold (actThre) and for all the actuators (na).

In order to take into account the minimization of the operational energy, the design process described in eqs.(1-10) is repeated iteratively within an outer loop. The main variable of the outer loop is the parameter MUT (material utilization factor). The active-passive system that corresponds to the minimum of the sum of embodied and operational energy is the optimum sought (fig.9).

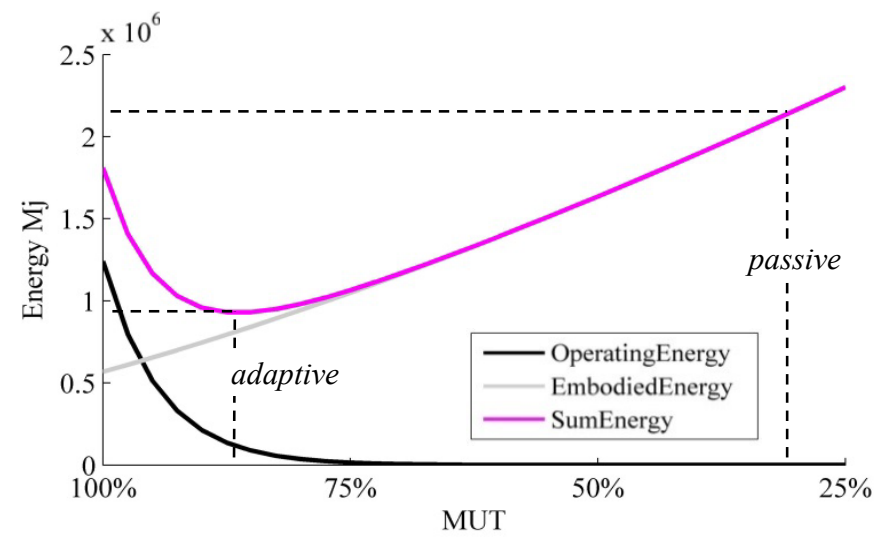

Figure 9 embodied \& operational energy curves

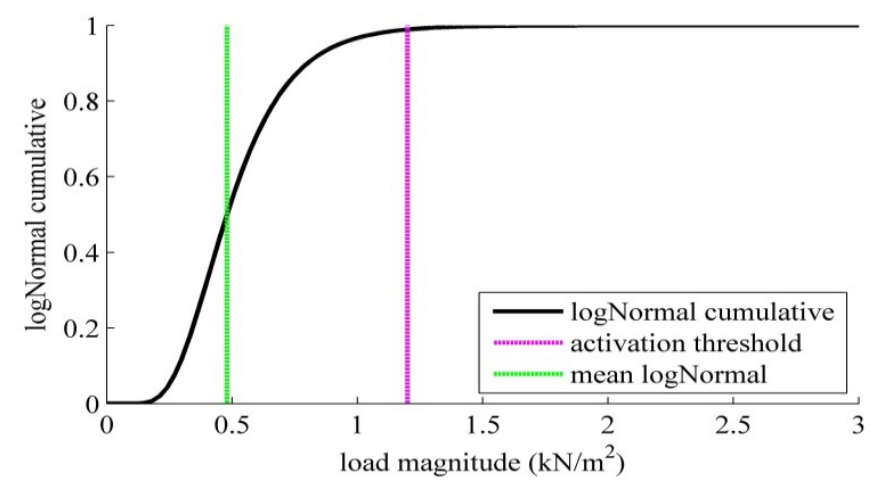

Figure 10 load activation threshold (LAT)

Fig. 10 shows the activation threshold that corresponds to the optimal MUT value. Comparison between the adaptive and an analogous structure optimized using the same formulation of eqs.(1-4) with additional compatibility constraints $\mathbf{K} \cdot \mathbf{u}_{\mathrm{k}}=\mathbf{P}$ shows that total energy savings remain substantial (55\% in this case) even when considering the operational energy (fig. 11).

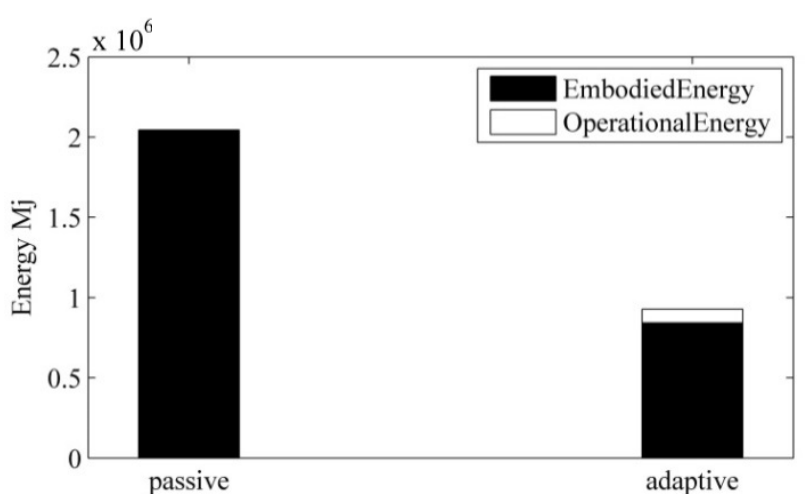

Figure 11 Energy savings comparison 
In both cases, embodied energy savings represent the major benefit when taken over the whole life of the structure, with other significant benefits in size reduction and construction transport and erection weight demands. The embodied energy is computed using the Bath Inventory of carbon and energy (Hammond \& Jones, 2008).

\subsection{Modifying the method for determinate cases}

This section studies the behavior of the determinate version of the cantilever truss described in the previous section (Fig. 12).

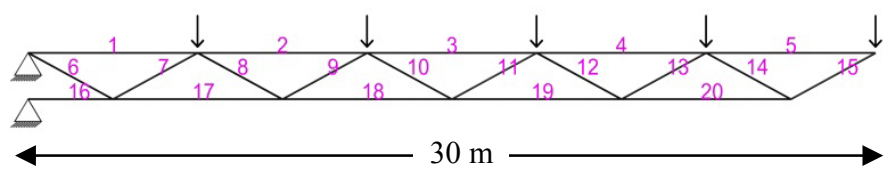

Figure 12 Determinate cantilever truss

The only difference in the mathematical formulation is in the computation of the actuators topology. For the determinate case the actuators length change does not change the state of stress of the structure thus eq. (7) and eq. (11) are not necessary. The problems in eq. (6) and eq. (10) can be solved directly by computing the pseudo-inverse of the sensitivity matrix for displacements:

$$
\Delta L=S^{U+} \cdot \Delta u
$$

which gives the actuators length change that compensate for displacements. Applying eqs.(8-11) gives the optimum actuator configuration for the displacements compensation. This is shown in Fig. 13.

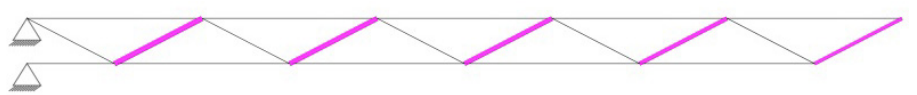

Figure 13 Optimal actuators topology determinate

Displacement correction can be dealt with in several ways. The simplest consists in setting the desired nodal displacement so as to stay within serviceability limits. A better way is to assign the desired displacement by taking into account the rate of change of the curvature between consecutive bays to avoid the formation of kinks in the deflection. Fig.14 shows the difference of the controlled shape with and without curvature constraints.

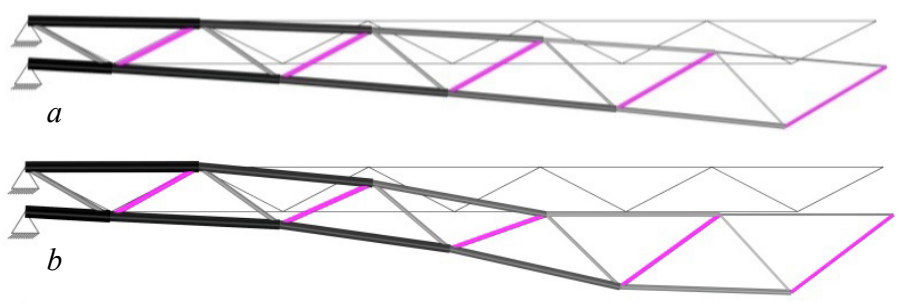

Figure 14 (a) curvature constr. (b) no curvature constr.

Comparison with an equivalent optimised passive truss gives energy savings of $60 \%$. Optimal forces at maximum expected load for the adaptive and passive configuration are shown in Fig.15.

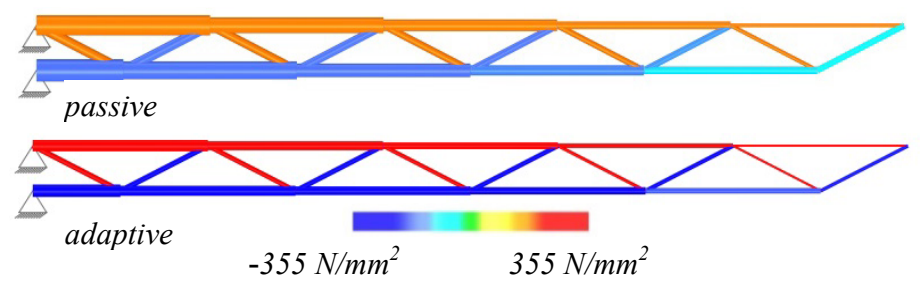

Figure 15 optimal stress distribution passive vs. adaptive

\section{DETERMINATE VS INDETERMIANTE}

In this section determinate and indeterminate trusses are compared in order to assess the effect of the load path redirection by the actuators for the control of internal forces and displacements. The next case study focuses on a simply supported truss beam supported by a pin at the bottom left corner and a roller at the bottom right node (Fig.16). The dead load is again $3 \mathrm{kN} / \mathrm{m}^{\wedge} 2$ supporting $10 \mathrm{~m}$ of roof out of plane. Two cases of live load are considered: in the first, a UDL is applied over the whole top cord whereas for the second only on the right half of the truss. The magnitude of the first UDL is set to dead load for the first load case and to twice the dead load for the second. The same load probability distribution as previously is taken for both the load cases

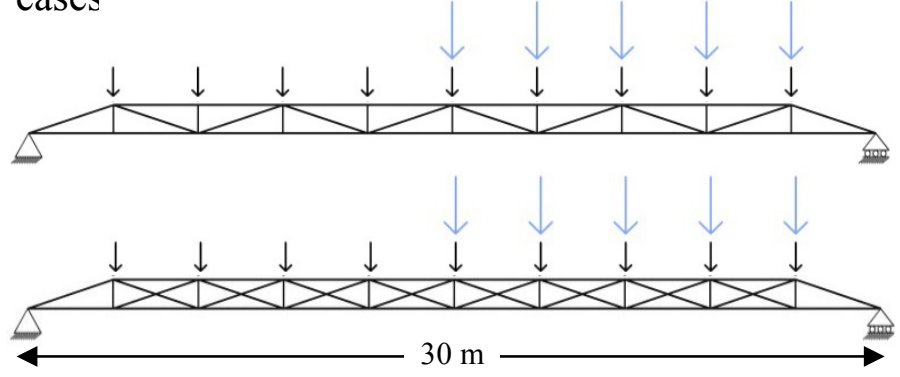

Figure 16 determinate \& indeterminate simply supported

The result of the optimization routine in terms of sections and actuators topology, only taking into account the first load case, are shown in Fig.17.

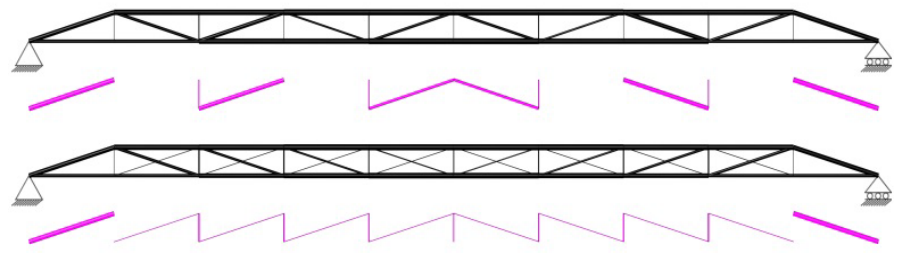

Figure 17 Adaptive trusses with actuators positions

The optimal forces for both determinate and indeterminate adaptive/passive designs are shown in Fig. 18. For the determinate case the axial forces in the adaptive and passive structures are simply scaled version of each other. The material is more efficiently utilized and stresses tend to reach the maximum allowable values in the active structure. 
determinate

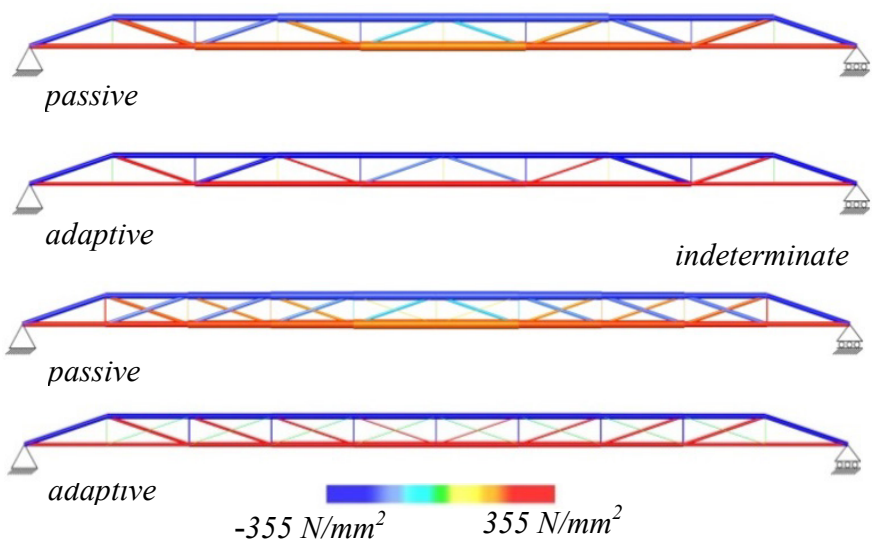

Figure 18 Comparison force distributions determinate vs. indeterminate case

By contrast, in the indeterminate case the axial forces in the passive and active truss are different. In the passive truss, the vertical elements work very little whereas the cross bracings are quite heavily used both in tension and in compression. The active indeterminate structure has all its vertical members in compression and in the cross bracing, the tension members have become dominant. Actuation has effectively turned the truss structure into a Pratt truss which is the best topology for the type of load acting on it.

In terms of total energy savings, both determinate and indeterminate designs achieve $50 \%$ with respect to the passive structures. Breaking down the total energy into embodied and operational shows that the adaptive indeterminate achieves $10 \%$ reduction for the former but a $40 \%$ increase for the latter compared to the adaptive determinate (Fig.19). The increase in operational energy is due to the load path redirection and the bigger number of actuators (Fig. 19).

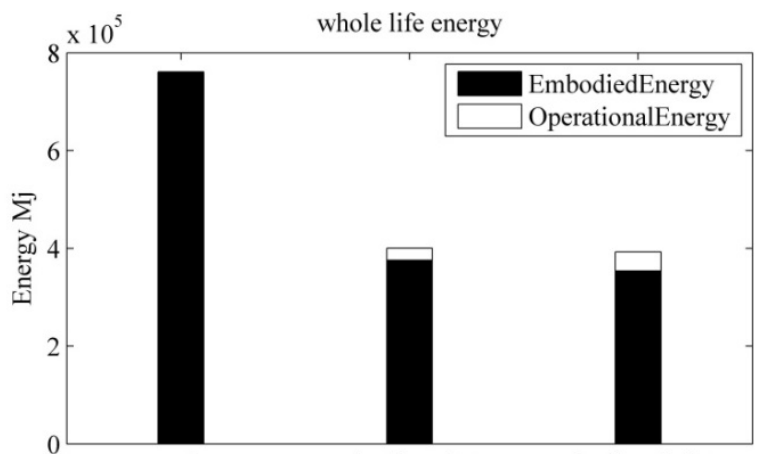

Figure 19 comparison passive vs det. \& indet. trusses

Similar results are obtained when the two load cases are applied as combination case (fig. 20).

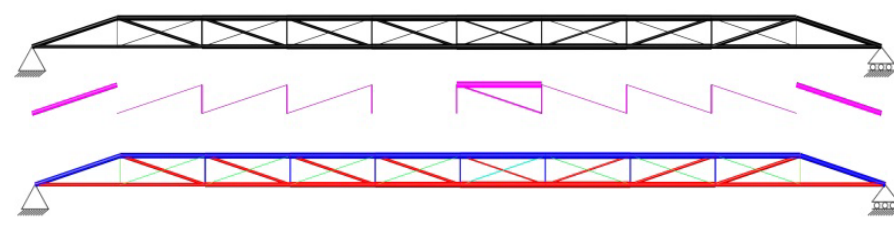

Figure 20 Force-sections distribution and actuators positions found for the load combination case.

\section{CONCLUSIONS}

A design procedure was presented that allows the design of structures that minimize whole life energy by introducing actuations at optimum placement in pin-jointed truss structures. Maximum energy savings are achieved by embedding the actuators with optimum placement and by finding the load threshold at which active control becomes necessary. Results from two planar structure case studies were presented. First for a determinate cantilever it was shown that the optimal active design allowed $50 \%$ of the total energy to be saved compared to an optimized passive design with the same topology. The second structure studied was a simply truss beam. Both a determinate and an indeterminate topology were tested. Similar savings around 50\% were achieved for both. The indeterminate truss allows load path redirection. This in principle could allow a better homogenization of the stresses inside the truss. However, the whole life energy costing showed that the extra actuation needed in the indeterminate truss allows only minor further substantial energy savings with respect to the determinate case.

\section{ACKNOWLEDGEMENTS}

The authors would like to thanks the EPSRC and Expedition Engineering for funding this research project.

\section{BIBLIOGRAPHY}

Hammond, G. \& Jones, C., 2008. Embodied Energy \& Carbon, Bath: University of Bath.

Nocedal, J. \& Wright, S. J., 2006. Numerical Optimization. s.l.:Springer Verlag.

Patnaik, S., Hopkins, D. \& Halford, G., 1991. Integrated Force Method versus Displacement Method for Finite Element Analysis. Computers and Structures, 38(4), pp. 377-407.

Ramrakhyani, D. S., Lesieutre, G. A., Frecker, M. \& \& Bharti, S., 2005. Aircraft Structural Morphing Using Tendon-Actuated Compliant Cellular Trusses. Journal of aircraft, 42(6), pp. 1615-1621.

Senatore, G. et al., 2011. Large Scale Adaptive Structures for Whole Life Energy Savings. International Association for Shell and Spatial Structures (IASS), 52(No. 4 December n. 170).

Spillers, W. \& MacBain, K., 2009. Structural Optimization. New York: Springer.

Teuffel, P., 2004. Entwerfen adaptiver Strukturen. http://elib.unistuttgart.de/opus/volltexte/2005/2172/pdf/ Teuffel_Entwerfen_Adaptiver_Strukturen.pdf 